\title{
Optimal Vibration Control for Tracked Vehicle Suspension Systems
}

\author{
Yan-Jun Liang ${ }^{1}$ and Shi-Liang $\mathrm{Wu}^{2}$ \\ ${ }^{1}$ School of Computer and Information Engineering, Anyang Normal University, Anyang 455000, China \\ ${ }^{2}$ School of Mathematics and Statistics, Anyang Normal University, Anyang 455000, China \\ Correspondence should be addressed to Yan-Jun Liang; myluck0404@126.com
}

Received 16 October 2013; Revised 5 December 2013; Accepted 5 December 2013

Academic Editor: Weichao Sun

Copyright ( 2013 Y.-J. Liang and S.-L. Wu. This is an open access article distributed under the Creative Commons Attribution License, which permits unrestricted use, distribution, and reproduction in any medium, provided the original work is properly cited.

Technique of optimal vibration control with exponential decay rate and simulation for vehicle active suspension systems is developed. Mechanical model and dynamic system for a class of tracked vehicle suspension vibration control is established and the corresponding system of state space form is described. In order to prolong the working life of suspension system and improve ride comfort, based on the active suspension vibration control devices and using optimal control approach, an optimal vibration controller with exponential decay rate is designed. Numerical simulations are carried out, and the control effects of the ordinary optimal controller and the proposed controller are compared. Numerical simulation results illustrate the effectiveness of the proposed technique.

\section{Introduction}

The vibration caused by severe pavement conditions not only affects the normal use of vehicle-mounted instruments but also damages the instruments, reduces ride ability, and even threatens the safety of passengers and vehicles. Therefore, the vibration control of the vehicle suspension is necessary.

Since people put forward higher requirements on the ride comfort and the operational stability, the passive suspension can not meet the current needs. However, the active suspension has outstanding advantage in the riding comfort and operational stability. Therefore, the active suspension vibration control technique has attracted many scholars' research interests. To improve suspension performance on driver ride comfort, integrated seat and suspension models and driver body models were investigated $[1,2]$, in which the problems of vibration control were studied. A nonlinear mathematical model of the dynamic suspension system with two degrees of freedom was developed and PID controller was designed for a kind of air suspension system in [3]. An active suspension system utilizing a low-cost high-performance linear switched reluctance actuator with proportional-derivative control was presented in [4]. To study the vibration control problems of the vehicle suspension, $H_{\infty}$ control theory was applied [59], in which robust $H_{\infty}$ control and linear matrix inequality optimization method, nonfragile $H_{\infty}$ control method, and adaptive neurofuzzy inference system inverse magnetorheological damper model were used, respectively. Sliding mode control strategy such as self-organizing fuzzy sliding-mode control and adaptive sliding-mode control methods were utilized in [10-12] to obtain vibration controllers for vehicle suspension systems. Considering structured uncertainties and unstructured uncertainties, adaptive robust controllers were designed in $[13,14]$.

The response time of the controller, namely, the real-time of the controller, is not considered in most of the research on vibration control of active suspension. In this paper, considered control energy and response time, used optimal control approach, optimal vibration control for tracked vehicle suspension systems is studied. The model of active suspension for tracked vehicle is established, optimal vibration controller with exponential decay rate is designed, and the control effect is demonstrated by numerical simulations.

The remainder of this paper is structured as follows. In Section 2, we establish active suspension systems and disturbance model. In Section 3, optimal vibration controller 


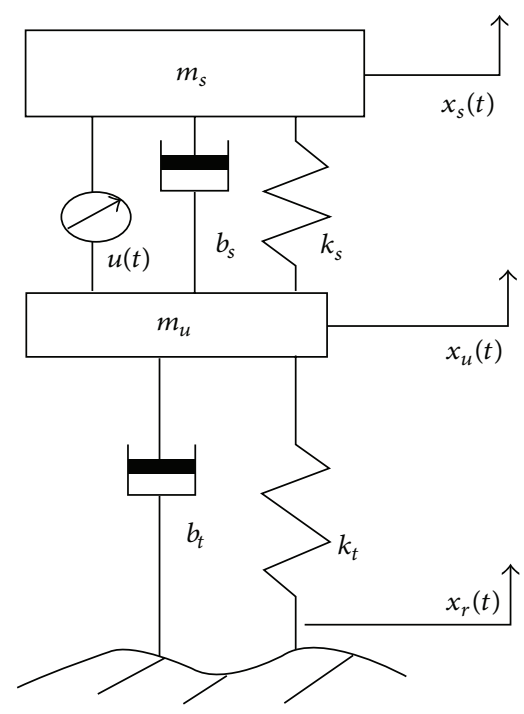

FIGURE 1: Quarter-car model with active suspension.

is designed for the suspension systems. In Section 4, numerical experiments are presented. Finally, in Section 5 some conclusions are drawn.

\section{Suspension Systems and Disturbance Model}

2.1. Mechanical Model of Suspension. Vehicle suspensions are complex dynamic systems, and the responses of suspensions are influenced by many factors. The suspension systems of four-wheel drive and four-wheel steering vehicles, strictly speaking, are eight degrees of freedom vibration systems. Based on practical problem and principle of being sufficient to analyze and study the vibration control for vehicle suspensions, the mechanical model of quarter-car suspension system with two degrees of freedom is investigated in this paper, as shown in Figure 1 (see $[15,16])$, in which $m_{s}$ is the sprung mass representing the tracked car chassis; $m_{u}$ is the unsprung mass representing the wheel assembly; $k_{s}$ and $b_{s}$ are stiffness and damping of the uncontrolled suspension, respectively; $k_{t}$ and $b_{t}$ stand for stiffness and damping of the wheel, respectively; $x_{s}(t)$ and $x_{u}(t)$ are the displacements of the sprung and unsprung masses, respectively; $x_{r}(t)$ is the road displacement input; $u(t)$ denotes the actuator control force, which is normally generated by an actuator between the two masses.

2.2. Dynamic Model of Suspension. Consider the quarter-car suspension system with two degrees of freedom as shown in Figure 1; the dynamic equations of $m_{s}$ and $m_{u}$ are given by

$$
\begin{aligned}
u(t)= & m_{s} \ddot{x}_{s}(t)+b_{s}\left[\dot{x}_{s}(t)-\dot{x}_{u}(t)\right]+k_{s}\left[x_{s}(t)-x_{u}(t)\right], \\
u(t)= & -m_{u} \ddot{x}_{u}(t)+b_{s}\left[\dot{x}_{s}(t)-\dot{x}_{u}(t)\right]+k_{s}\left[x_{s}(t)-x_{u}(t)\right] \\
& -k_{t}\left[x_{u}(t)-x_{r}(t)\right]-b_{t}\left[\dot{x}_{u}(t)-\dot{x}_{r}(t)\right]
\end{aligned}
$$

where $\ddot{x}_{s}(t)$ and $\dot{x}_{s}(t)$, respectively, are acceleration and velocity of the sprung and unsprung masses; $\ddot{x}_{u}(t)$ and $\dot{x}_{u}(t)$, respectively, are acceleration and velocity of the unsprung masses; $\dot{x}_{r}(t)$ is the road velocity.

Define the set of state variables for (1):

$$
\begin{aligned}
& x_{1}(t)=x_{s}(t)-x_{u}(t), \\
& x_{2}(t)=x_{u}(t)-x_{r}(t), \\
& x_{3}(t)=\dot{x}_{s}(t), \\
& x_{4}(t)=\dot{x}_{u}(t),
\end{aligned}
$$

where $x_{1}(t)$ is the suspension deflection, $x_{2}(t)$ is the wheel deflection, $x_{3}(t)$ is the sprung mass velocity, and $x_{4}(t)$ is the unsprung mass velocity. Then, the state vector is in the following form:

$$
x(t)=\left[x_{1}(t), x_{2}(t), x_{3}(t), x_{4}(t)\right]^{T} .
$$

The principal variables for the active suspension design and evaluation are sprung mass acceleration $\ddot{x}_{s}(t)$, which determines the ride comfort; the suspension deflection $x_{s}(t)-$ $x_{u}(t)$, which indicates the limit of the vehicle body motion; and the wheel deflection $x_{u}(t)-x_{r}(t)$, which ensures the road holding ability. To satisfy the performance requirements, the controlled output vector is chosen as

$$
y_{c}(t)=\left[\begin{array}{c}
\ddot{x}_{s}(t) \\
x_{s}(t)-x_{u}(t) \\
x_{u}(t)-x_{r}(t)
\end{array}\right]=\left[\begin{array}{c}
\ddot{x}_{s}(t) \\
x_{1}(t) \\
x_{2}(t)
\end{array}\right] .
$$

Then, from motion equation (1), state variables (2), state vector (3), and output vector (4), the dynamic system model of vehicle suspension is rewritten in the state-space representation:

$$
\begin{gathered}
\dot{x}(t)=A x(t)+B u(t)+D p(t), \\
y_{c}(t)=\bar{C} x(t)+\bar{B} u(t), \\
x(0)=x_{0},
\end{gathered}
$$

where $p(t)=\dot{x}_{r}(t)$ is external input disturbance, and

$$
A=\left[\begin{array}{cccc}
0 & 0 & 1 & -1 \\
0 & 0 & 0 & 1 \\
\frac{-k_{s}}{m_{s}} & 0 & \frac{-b_{s}}{m_{s}} & \frac{b_{s}}{m_{s}} \\
\frac{k_{s}}{m_{u}} & \frac{-k_{t}}{m_{u}} & \frac{b_{s}}{m_{u}} & \frac{-\left(b_{t}+b_{s}\right)}{m_{u}}
\end{array}\right],
$$

$$
\begin{gathered}
B=\left[\begin{array}{c}
0 \\
0 \\
\frac{1}{m_{s}} \\
\frac{-1}{m_{u}}
\end{array}\right], \quad D=\left[\begin{array}{c}
0 \\
-1 \\
0 \\
\frac{b_{t}}{m_{u}}
\end{array}\right], \quad \bar{B}=\left[\begin{array}{c}
\frac{1}{m_{s}} \\
0 \\
0
\end{array}\right], \\
\bar{C}=\left[\begin{array}{cccc}
\frac{-k_{s}}{m_{s}} & 0 & \frac{-b_{s}}{m_{s}} & \frac{b_{s}}{m_{s}} \\
1 & 0 & 0 & 0 \\
0 & 1 & 0 & 0
\end{array}\right] .
\end{gathered}
$$


TABLE 1: Road grades and PSDs.

\begin{tabular}{lccccc}
\hline Road grade & A & B & C & D & E \\
\hline$C_{s}\left(10^{-7} \mathrm{~m}^{3} / \mathrm{rad}\right)$ & 1 & 4 & 16 & 64 & 256 \\
Road sort $k$ & 0 & 1 & 2 & 3 & 4 \\
\hline
\end{tabular}

2.3. Disturbance Model. To evaluate the suspension characteristics, the road profiles variability is taken into account. According to the ISO 2631 standards, the road displacement power spectral density (PSD) is approximately represented in the formulation of

$$
S\left(W_{1}\right)=C_{s} W_{1}^{-2}=4^{k} \times 10^{-7} W_{1}^{-2},
$$

where $W_{1}$ is the spatial frequency, $C_{s}$ is the road roughness constant, and $k$ denotes the sorts of road grade as shown in Table 1.

Assume that the vehicle travels at a certain constant horizontal velocity $v_{0}$ and the given road segment length is $l$. The road disturbances are approximately considered as periodic vibrations. Since vehicle wheels and the active suspension system have the low pass filter characteristic, the road displacement $x_{r}(t)$ can be approximately simulated by a finite series sum

$$
x_{r}(t)=\sum_{j=1}^{i} \xi_{j}(t) \triangleq \sum_{j=1}^{i} \phi_{j} \sin \left(j \omega_{0} t+\theta_{j}\right)
$$

with amplitudes $\phi_{j}$ and random phases $\theta_{j}$ which follows a uniform distribution in $[0,2 \pi]$. According to the random process theory, the $j$ th average power is as follows:

$$
S\left(j \Delta W_{1}\right) \times \Delta W_{1}=\frac{\phi_{j}^{2}}{2} .
$$

Hence, we get

$$
\phi_{j}=\sqrt{2 S\left(j \Delta W_{1}\right) \Delta W_{1}}=\frac{2^{k}}{10^{3} j} \sqrt{\frac{l}{10 \pi}} .
$$

Choose the spatial frequency interval $\Delta W_{1}=2 \pi / l$ and then the time frequency internal $\omega_{0}=2 \pi v_{0} / l$. Positive integer $i$ limits the considered frequency band, and in this simplified model, it is generally lower than $20 \mathrm{~Hz}$.

Defining the disturbance state vector,

$$
\begin{aligned}
w(t) & =\left[w_{1}(t), w_{2}(t), \ldots, w_{2 i}(t)\right]^{T} \\
& =\left[\xi_{1}(t), \ldots, \xi_{i}(t), \dot{\xi}_{1}(t), \ldots, \dot{\xi}_{i}(t)\right]^{T} .
\end{aligned}
$$

The road velocity disturbance $p(t)=\dot{x}_{r}(t)$ is described by the following exosystem:

$$
\begin{aligned}
& \dot{w}(t)=G w(t), \\
& p(t)=F w(t),
\end{aligned}
$$

where

$$
\begin{gathered}
G=\left[\begin{array}{cc}
0 & I_{i} \\
-\bar{\Omega}^{2} & 0
\end{array}\right] \in \mathbb{R}^{2 i \times 2 i}, \\
F=[\underbrace{1, \ldots, 1,}_{i} \underbrace{0, \ldots, 0}_{i}] \in \mathbb{R}^{1 \times 2 i},
\end{gathered}
$$

in which $\bar{\Omega}=\operatorname{diag}\left\{\omega_{0}, 2 \omega_{0}, \ldots, i \omega_{0}\right\} \in \mathbb{R}^{i \times i}$. Evidently, the pair $(F, G)$ is observable completely.

\section{Optimal Vibration Controller Design}

In order to study optimal vibration control problem for tracked vehicles, we choose an average performance index for system (5) as follows:

$$
J=\lim _{T \rightarrow \infty} \frac{1}{T} \int_{0}^{T} e^{2 \alpha t}\left[x^{T}(t) Q x(t)+u^{T}(t) R u(t)\right] d t
$$

where $Q=C^{T} C \in \mathbb{R}^{4 \times 4}$ are positive semidefinite matrices, $R \in \mathbb{R}$ is a positive definite matrix, and $\alpha \geq 0$ is exponential decay rate, and if $\alpha=0$, it is an ordinary optimal control performance index. We can modify the values of $\alpha, Q$, and $R$ to satisfy the control purpose, such as the response time of the control system and the balance between the control energy and control effect.

The objective of this paper is to find a control law for system (5) and make the value of the performance index (14) minimum.

Then, we design vibration controller for system (5). The optimal control law with exponential decay rate can be presented in the following theorem.

Theorem 1. Consider the optimal control problem described by system (5) with performance index (14); the optimal control law exists and is unique. Its form is as follows:

$$
u^{*}(t)=-R^{-1} B^{T}\left[P_{1} x(t)+P_{2} p(t) e^{-\alpha t}+P_{3} p_{\omega}(t) e^{-\alpha t}\right],
$$

where $P_{1}$ is the unique positive definite solution of the following Riccati matrix equation:

$$
(A+\alpha I)^{T} P_{1}+P_{1}(A+\alpha I)-P_{1} S P_{1}+Q=0,
$$

where $P_{2}$ and $P_{3}$ are the unique solutions of the following Sylvester matrix equations:

$$
\begin{gathered}
{\left[(A+\alpha I)^{T}-P_{1} S\right]^{2} P_{2}+P_{2} \Omega^{2}=-\left[(A+\alpha I)^{T}-P_{1} S\right] P_{1} D} \\
{\left[(A+\alpha I)^{T}-P_{1} S\right]^{2} P_{3}+P_{3} \Omega^{2}=P_{1} D}
\end{gathered}
$$

in which $S=R^{-1} B^{T}, \Omega=\operatorname{diag}\{\bar{\Omega}, \bar{\Omega}\}, p_{\omega}(t)=\dot{p}(t)=F G w(t)$.

Proof. Introduce model transform for the system (5) with performance index (14):

$$
\begin{gathered}
\widetilde{x}(t)=x(t) e^{\alpha t}, \quad \widetilde{u}(t)=u(t) e^{\alpha t}, \quad \widetilde{p}(t)=p(t), \\
\widetilde{A}=A+\alpha I, \quad \widetilde{B}=B, \quad \widetilde{D}=D .
\end{gathered}
$$


Then we have

$$
\begin{aligned}
\dot{\tilde{x}}(t) & =\dot{x}(t) e^{\alpha t}+\alpha x(t) e^{\alpha t} \\
& =A x(t) e^{\alpha t}+\alpha x(t) e^{\alpha t}+B u(t) e^{\alpha t}+D p(t) e^{\alpha t} \\
& =\widetilde{A} \tilde{x}(t)+\widetilde{B} \tilde{u}(t)+\widetilde{D} \widetilde{p}(t), \\
J & =\lim _{T \rightarrow \infty} \frac{1}{T} \int_{0}^{T} e^{2 \alpha t}\left[x^{T}(t) Q x(t)+u^{T}(t) R u(t)\right] d t \\
& =\lim _{T \rightarrow \infty} \frac{1}{T} \int_{0}^{T}\left[\left(x(t) e^{\alpha t}\right)^{T} Q\left(x(t) e^{\alpha t}\right)\right. \\
& \left.\quad+\left(u(t) e^{\alpha t}\right)^{T} R\left(u(t) e^{\alpha t}\right)\right] d t \\
& =\lim _{T \rightarrow \infty} \frac{1}{T} \int_{0}^{T}\left[\tilde{x}^{T}(t) Q \tilde{x}(t)+\widetilde{u}^{T}(t) R \widetilde{u}(t)\right] d t .
\end{aligned}
$$

Applying the maximum principle to the optimal control problem in (20) and (21), the optimal control law can be written as

$$
\widetilde{u}^{*}(t)=-R^{-1} \widetilde{B}^{T} \widetilde{\lambda}(t)
$$

where $\widetilde{\lambda}(t)$ is the solution to the following two-point boundary value problem:

$$
\begin{gathered}
-\dot{\tilde{\lambda}}(t)=Q \dot{\tilde{x}}(t)+A^{T} \widetilde{\lambda}(t), \\
\dot{\tilde{x}}(t)=A \widetilde{x}(t)-R^{-1} \widetilde{B}^{T} \widetilde{\lambda}(t)+D \widetilde{p}(t), \\
\tilde{x}(0)=x_{0} e^{\alpha t} \\
\tilde{\lambda}(\infty)=0 .
\end{gathered}
$$

To solve (23), let

$$
\tilde{\lambda}(t)=P_{1} \tilde{x}(t)+P_{2} \tilde{p}(t)+P_{3} \widetilde{p}_{\omega}(t) .
$$

Substituting the equations of (20) and (22) into the first derivatives of (24), we get

$$
\begin{aligned}
\dot{\tilde{\lambda}}(t)= & P_{1} \dot{\tilde{x}}(t)+P_{2} \dot{\tilde{p}}(t)+P_{3} \ddot{\tilde{p}}(t) \\
= & \left(P_{1} \widetilde{A}-P_{1} S P_{1}\right) \tilde{x}(t)+\left(P_{1} \widetilde{D}-P_{1} S P_{2}\right) \tilde{p}(t) \\
& +\left(P_{2}-P_{1} S P_{3}\right) \dot{\tilde{p}}(t)+P_{3} \ddot{\widetilde{p}}(t) .
\end{aligned}
$$

Note that

$$
\begin{aligned}
\widetilde{p}_{\omega}(t) & =p_{\omega}(t)=\dot{p}(t), \\
\ddot{\tilde{p}}(t) & =\ddot{p}(t)=G^{2} p(t) \\
& =-\left[\begin{array}{cc}
\bar{\Omega}^{2} & 0 \\
0 & \bar{\Omega}^{2}
\end{array}\right] p(t) \\
& =-\Omega^{2} p(t) .
\end{aligned}
$$

From (23) and (24), we obtain

$$
\dot{\tilde{\lambda}}(t)=-\left(Q+\widetilde{A}^{T} P_{1}\right) \tilde{x}(t)-\widetilde{A}^{T} P_{2} \widetilde{p}(t)-\widetilde{A}^{T} P_{3} \widetilde{p}_{\omega}(t) .
$$

Adding (26) into (25), then comparing the coefficients of (25) and (27), we obtain matrix equations:

$$
\begin{gathered}
\widetilde{A}^{T} P_{1}+P_{1} \widetilde{A}-P_{1} S P_{1}+Q=0, \\
\widetilde{A}^{T} P_{2}+P_{1} \widetilde{D}-P_{3} \Omega^{2}-P_{1} S P_{2}=0, \\
\widetilde{A}^{T} P_{3}+P_{2}-P_{1} S P_{3}=0 .
\end{gathered}
$$

Then, adding the model transform (18), we can obtain

$$
\begin{aligned}
& (A+\alpha I)^{T} P_{1}+P_{1}(A+\alpha I)-P_{1} S P_{1}+Q=0 \\
& {\left[(A+\alpha I)^{T}-P_{1} S\right]^{2} P_{2}+P_{2} \Omega^{2}=-\left[(A+\alpha I)^{T}-P_{1} S\right] P_{1} D} \\
& {\left[(A+\alpha I)^{T}-P_{1} S\right]^{2} P_{3}+P_{3} \Omega^{2}=P_{1} D}
\end{aligned}
$$

In the following, we prove the existence and uniqueness of the optimal control law. In fact, it is equivalent to prove the existence and uniqueness of the matrices $P_{1}, P_{2}$, and $P_{3}$. It was well known that the matrix $P_{1}$ is existent and unique.

According to the linear regulator theory, it follows that

$$
\operatorname{Re} \lambda_{i}\left[(A+\alpha I)^{T}-P_{1} S\right]<-\alpha<0, \quad i=1,2, \ldots, n \text {. }
$$

Suppose that

$$
\lambda_{i}\left[(A+\alpha I)^{T}-P_{1} S\right]=-\alpha_{i}+j \beta_{i},
$$

where $\alpha_{i}>0, \beta_{i} \in R, j=\sqrt{-1}$. Therefore, we have

$$
\begin{aligned}
\lambda_{i}\left[(A+\alpha I)^{T}-P_{1} S\right]^{2} & =\left\{\lambda_{i}\left[(A+\alpha I)^{T}-P_{1} S\right]\right\}^{2} \\
& =\alpha_{i}^{2}-\beta_{i}^{2}-j 2 \alpha_{i} \beta_{i} .
\end{aligned}
$$

Due to $\Omega=\operatorname{diag}(\bar{\Omega}, \bar{\Omega})$, we obtain

$$
\lambda_{j}\left(-\Omega^{2}\right) \leq 0, \quad j=1,2, \ldots, 2 m .
$$

Assume that there is a certain $\lambda_{i}\left(A-S P_{1}\right)^{2}=\lambda_{j}\left(-\Omega^{2}\right)$; from (32) and (33), we have a certain $\alpha_{i}=0$ at least. It is contrary to $\alpha_{i}>0$. Namely, $\lambda_{i}\left(A-S P_{1}\right)^{2} \neq \lambda_{j}\left(-\Omega^{2}\right)$.

Hence, $P_{2}$ and $P_{3}$, the solution of Sylvester matrix (17), are existent and unique [17].

When the matrices $P_{1}, P_{2}$, and $P_{3}$ are derived, $\tilde{\lambda}(t)$ and the optimal control law $\widetilde{u}^{*}(t)$ can be obtained from (24) and (22), respectively. According to model transform (19), the optimal control law (15) is obtained.

\section{Numerical Simulations}

In this section, we apply the proposed optimal vibration controller to a tracked vehicle. The model parameters have 
TABle 2: Parameters of quarter-car model.

\begin{tabular}{lccc}
\hline Parameter & Variable & Value & Unit \\
\hline Sprung mass & $m_{s}$ & 972.2 & $\mathrm{~kg}$ \\
Unsprung mass & $m_{u}$ & 113.6 & $\mathrm{~kg}$ \\
Suspension stiffness & $k_{s}$ & $42,719.6$ & $\mathrm{~N} / \mathrm{m}$ \\
Wheel stiffness & $k_{t}$ & 101,115 & $\mathrm{~N} / \mathrm{m}$ \\
Suspension damping & $b_{s}$ & 1,095 & $\mathrm{~N} \cdot \mathrm{s} / \mathrm{m}$ \\
Wheel damping & $b_{t}$ & 14.6 & $\mathrm{~N} \cdot \mathrm{s} / \mathrm{m}$ \\
\hline
\end{tabular}

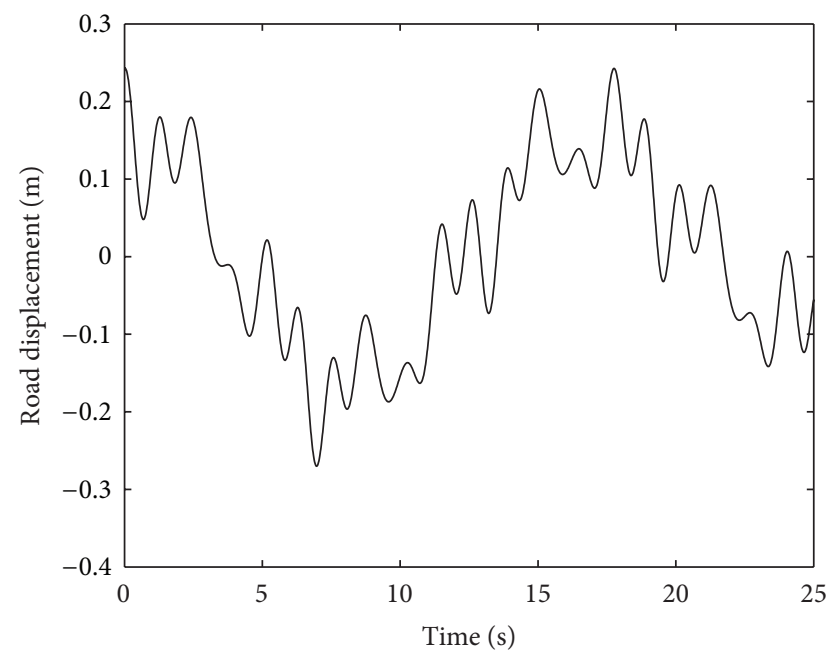

FIGURE 2: Road displacement curve.

the values listed in Table 2 which have been used in some references $[15,16]$. The associated matrices of system (5) are as follows:

$$
\begin{aligned}
& A=\left[\begin{array}{cccc}
0 & 0 & 1 & -1 \\
0 & 0 & 0 & 1 \\
-43.9412 & 0 & -1.1263 & 1.1263 \\
376.0528 & -890.0968 & 9.6391 & -9.7676
\end{array}\right] \text {, } \\
& B=\left[\begin{array}{c}
0 \\
0 \\
0.001 \\
-0.0088
\end{array}\right], \quad D=\left[\begin{array}{c}
0 \\
0 \\
0 \\
0.1285
\end{array}\right] \text {, } \\
& \bar{C}=\left[\begin{array}{cccc}
-43.9412 & 0 & -1.1263 & 1.1263 \\
1 & 0 & 0 & 0 \\
0 & 1 & 0 & 0
\end{array}\right], \quad \bar{B}=\left[\begin{array}{c}
0.001 \\
0 \\
0
\end{array}\right] \text {. }
\end{aligned}
$$

To generate $\mathrm{D}$ grade road profile, we select $C_{s}=64 \times$ $10^{-7} \mathrm{~m}^{3} / \mathrm{rad}$ and $k=3$ in Table 1 . Setting $v_{0}=20 \mathrm{~m} / \mathrm{s}, l=$ $400 \mathrm{~m}$, and $i=200 \mathrm{in}(8)$ and (10) takes the frequency band from $0.05 \mathrm{~Hz}$ to $10 \mathrm{~Hz}$.

Using MATLAB software, numerical experiment is carried out for the proposed optimal vibration controller. Figure 2 shows the road displacement curve.

The main purpose of vibration control of vehicle suspensions is to reduce sprung mass acceleration to enhance ride comfort, to reduce the suspension deflection which indicates

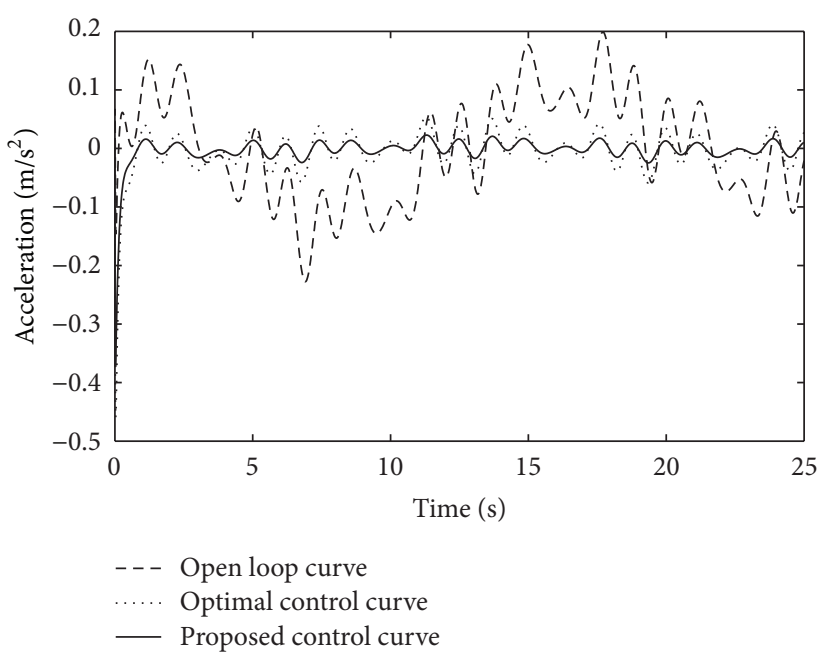

FIGURE 3: Sprung mass acceleration curves.

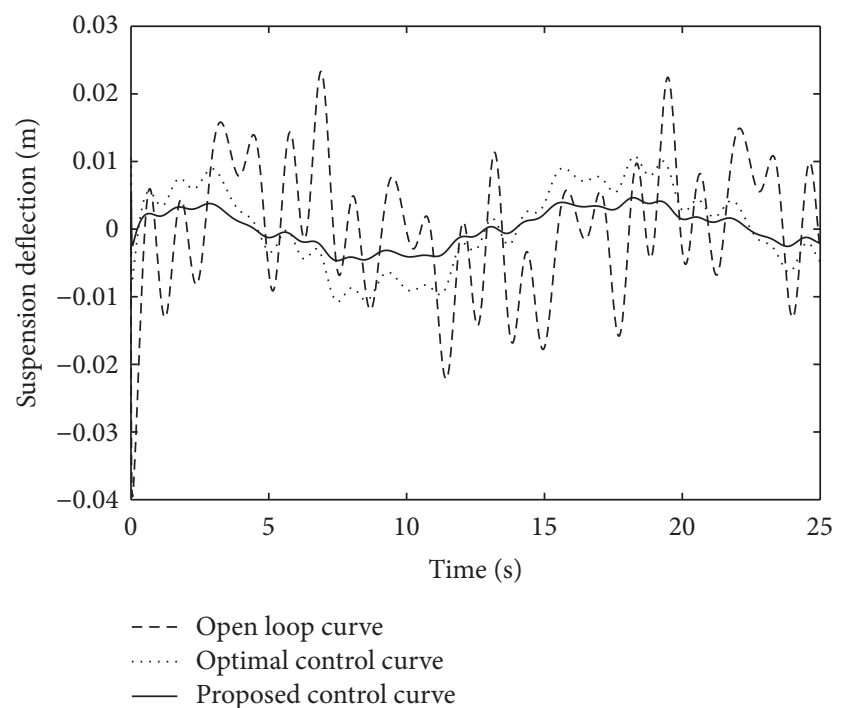

Figure 4: Suspension deflection curves.

the limit of the vehicle body motion, and to reduce the wheel deflection to ensure road holding ability. So, to evaluate effectiveness of the proposed control strategy, sprung mass acceleration, suspension deflection, and wheel defection are considered. In order to demonstrate the effect of the proposed optimal vibration controller $(\alpha=1)$, we compare it with other two control strategies: open loop and traditional optimal control $(\alpha=1)$, see Figures 3-6.

The curves of sprung mass acceleration $\ddot{x}_{s}(t)$ are shown in Figure 3, suspension deflections $x_{s}(t)-x_{u}(t)$ are shown in Figure 4, and wheel deflections $x_{u}(t)-x_{r}(t)$ are shown in Figure 5, in which dash lines represent the open loop results of the suspension systems, dotted lines represent the traditional optimal vibration control results, and solid lines describe the results of the suspension systems controlled by the proposed control strategy. It can be seen from these numerical results that the proposed optimal controller with 


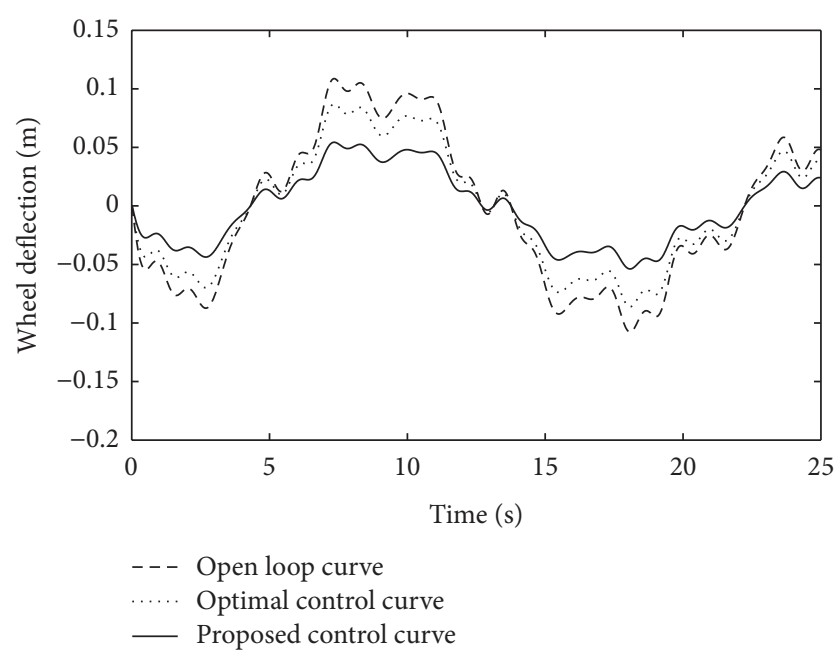

FIGURE 5: Wheel deflection curves.

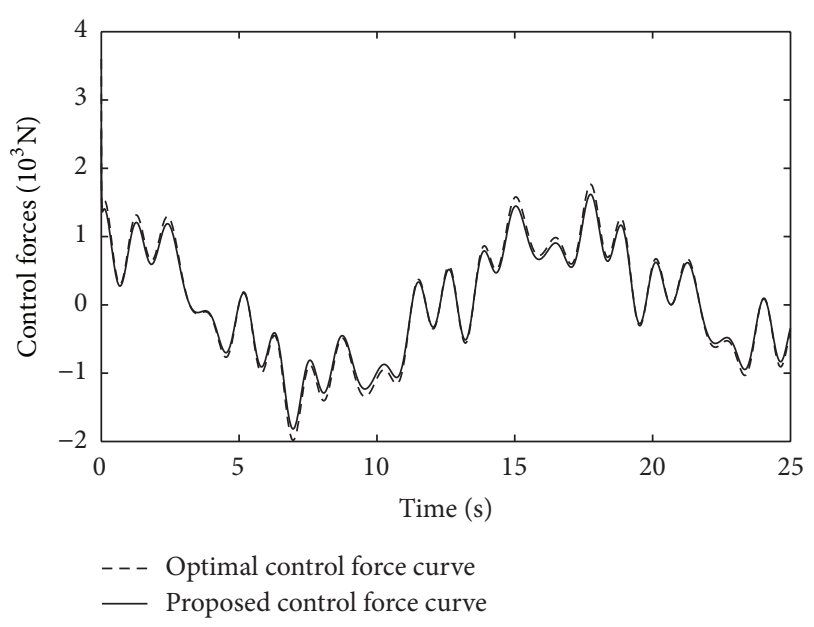

FIGURE 6: Control force curves.

exponential decay rate is efficient, real-time, and robust in reducing sprung mass acceleration and suspension deflection, thereby enhancing ride comfort and ensuring safety of passengers and vehicles. Moreover, in Figure 6, it demonstrates that it needs less control forces than traditional optimal vibration control; therefore, it needs less energy.

\section{Conclusions}

Suspension control plays an important role in the modern vehicle and is one of the very important components to provide the ride comfort, in particular, to reduce driver fatigue due to long hours of driving. In order to enhance the ride comfort and ensure the safety of passengers and vehicles, dynamic system for a class of tracked vehicle suspension vibration control is established and a kind of optimal controller with exponential decay rate is designed. Numerical simulations demonstrated that the proposed strategy is efficient, real-time, and robust.

\section{Acknowledgments}

The authors would like to thank the anonymous referees for their helpful suggestions, which greatly improve the paper. This research was supported by NSFC Tianyuan Mathematics Youth Fund (no. 11026040), the Nature Science Foundation of China (no. 11301009 and no. U1204402), Science \& Technology Development Plan of Henan Province (no. 122300410316), and Natural Science Foundations of Henan Province Education Department (no. 12A120001, no. 13A520018, and no. 13A110022).

\section{References}

[1] H. Du, W. Li, and N. Zhang, "Vibration control of vehicle seat integrating with chassis suspension and driver body model," Advances in Structural Engineering, vol. 16, pp. 1-10, 2013.

[2] H. Du, W. Li, and N. Zhang, "Integrated seat and suspension control for a quarter car with driver model," IEEE Transactions on Vehicular Technology, vol. 61, pp. 3893-3908, 2012.

[3] K. Prabu, J. Jancirani, J. Dennie, and B. Arun, "Vibrational control of air suspension system using PID controller," Journal of Vibroengineering, vol. 15, pp. 132-138, 2013.

[4] J. Lin, K. W. E. Cheng, Z. Zhang, N. C. Cheung, X. Xue, and T. W. Ng, "Active suspension system based on linear switched reluctance actuator and control schemes," IEEE Transactions on Vehicular Technology, vol. 62, pp. 562-572, 2013.

[5] L.-X. Guo and L.-P. Zhang, "Robust $H_{\infty}$ control of active vehicle suspension under non-stationary running," Journal of Sound \& Vibration, vol. 331, pp. 5824-5837, 2012.

[6] H. Li, H. Liu, C. Hilton, and S. Hand, "Non-fragile $H_{\infty}$ control for half-vehicle active suspension systems with actuator uncertainties," Journal of Vibration \& Control, vol. 19, pp. 560$575,2013$.

[7] L.-H. Zong, X.-L. Gong, S.-H. Xuan, and C.-Y. Guo, "Semiactive $H_{\infty}$ control of high-speed railway vehicle suspension with magnetorheological dampers," Vehicle System Dynamics, vol. 51, pp. 600-626, 2013.

[8] W. Sun, H. Gao, and O. Kaynak, "Finite frequency $H_{\infty}$ control for vehicle active suspension systems," IEEE Transactions on Control Systems Technology, vol. 19, no. 2, pp. 416-422, 2011.

[9] W. Sun, Y. Zhao, J. Li, L. Zhang, and H. Gao, "Active suspension control with frequency band constraints and actuator input delay," IEEE Transactions on Industrial Electronics, vol. 59, no. 1, pp. 530-537, 2012.

[10] R.-J. Lian, "Enhanced adaptive self-organizing fuzzy slidingmode controller for active suspension systems," IEEE Transactions on Industrial Electronics, vol. 60, pp. 958-968, 2013.

[11] H. Li, J. Yu, C. Hilton, and H. Liu, "Adaptive sliding-mode control for nonlinear active suspension vehicle systems using T$S$ fuzzy approach," IEEE Transactions on Industrial Electronics, vol. 60, pp. 3328-3338, 2013.

[12] J.-L. Yao, W.-K. Shi, J.-Q. Zheng, and H.-P. Zhou, "Development of a sliding mode controller for semi-active vehicle suspensions," Journal of Vibration \& Control, vol. 19, pp. 1152-1160, 2013.

[13] J. Yao, Z. Jiao, and D. Ma, "Adaptive robust control of DC motors with extended state observer," IEEE Transactions on Industrial Electronics, 2013. 
[14] J. Yao, Z. Jiao, D. Ma, and L. Yan, "High-accuracy tracking control of hydraulic rotary actuators with modeling uncertainties," IEEE/ASME Transactions on Mechatronics, vol. 99, pp. 1-9, 2013.

[15] H. Du and N. Zhang, " $H_{\infty}$ control of active vehicle suspensions with actuator time delay," Journal of Sound and Vibration, vol. 301, no. 1-2, pp. 236-252, 2007.

[16] N. Jalili and E. Esmailzadeh, "Optimum active vehicle suspensions with actuator time delay," Journal of Dynamic Systems, Measurement and Control, vol. 123, no. 1, pp. 54-61, 2001.

[17] S. K. Mitra, "The matrix equation $A \times B+C \times D=E$," SIAM Journal on Applied Mathematics, vol. 32, pp. 823-825, 1977. 


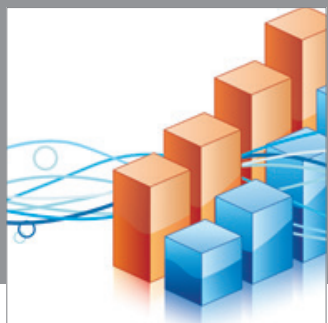

Advances in

Operations Research

mansans

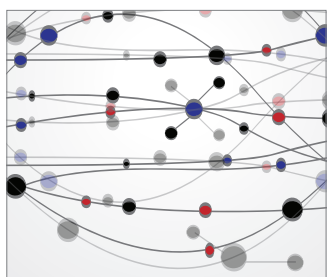

The Scientific World Journal
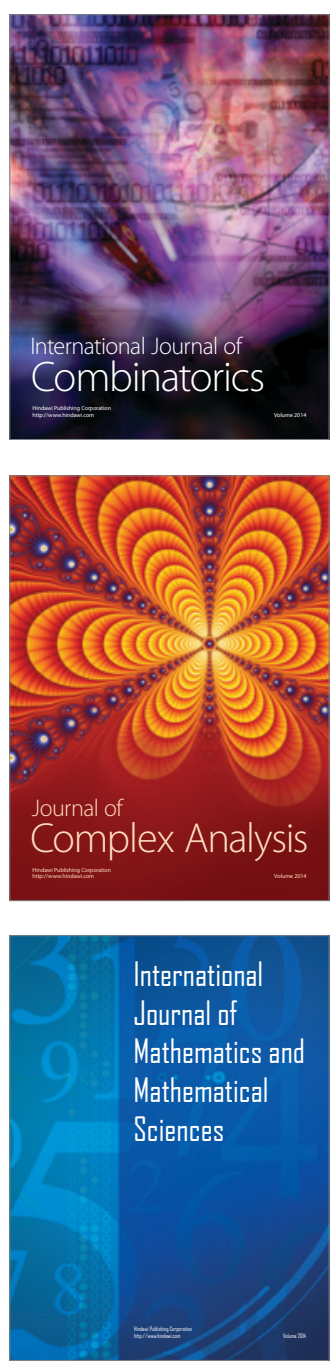
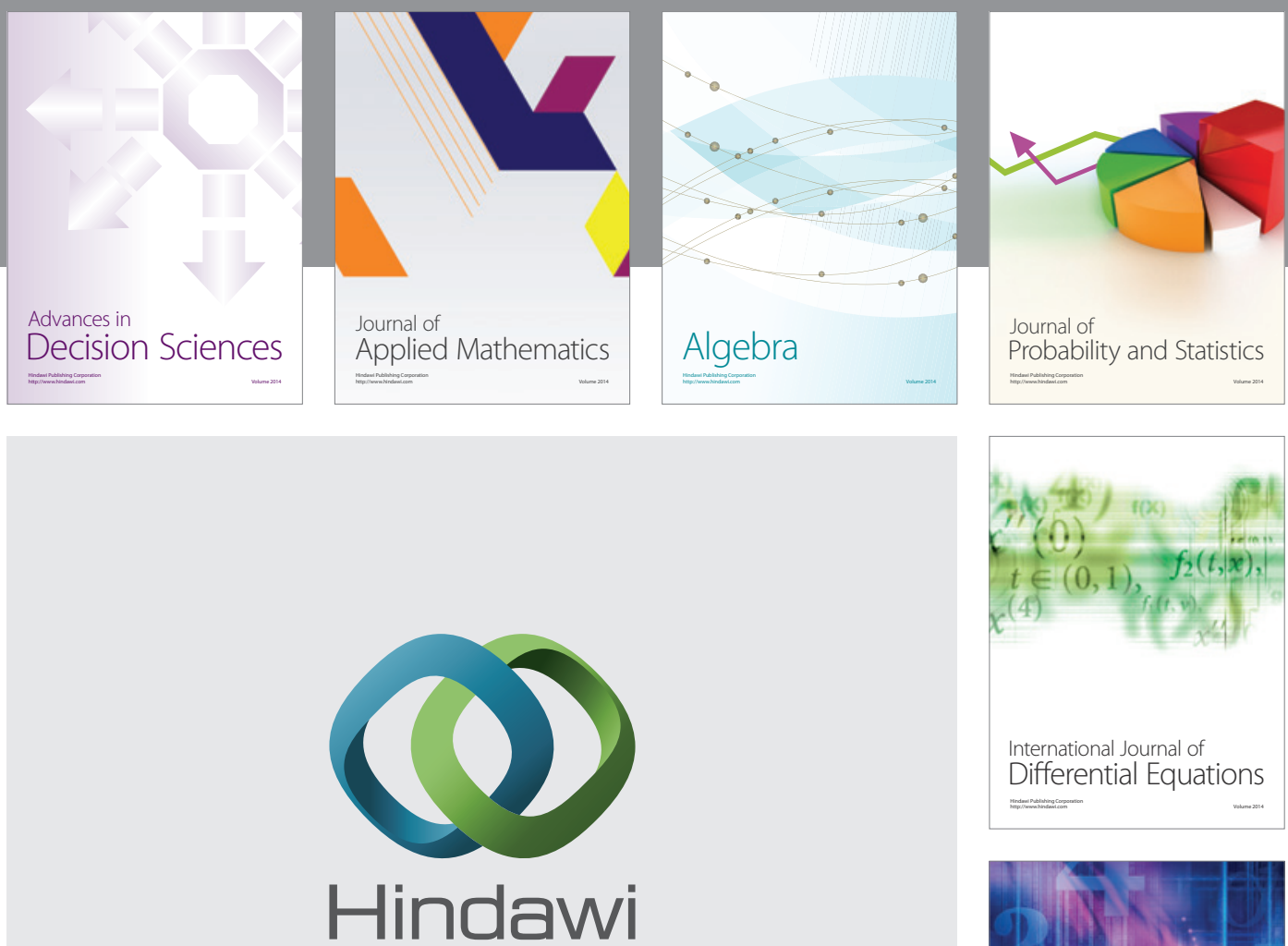

Submit your manuscripts at http://www.hindawi.com
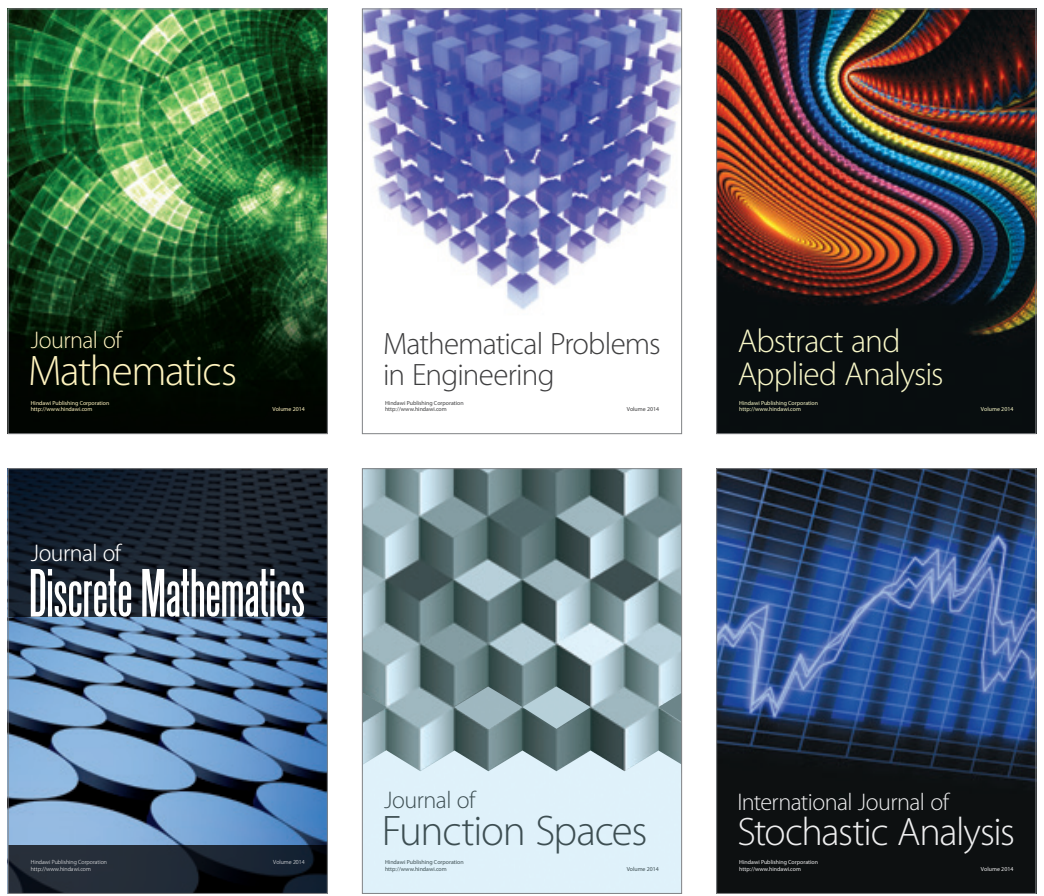

Journal of

Function Spaces

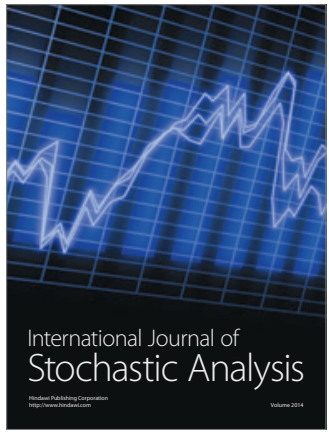

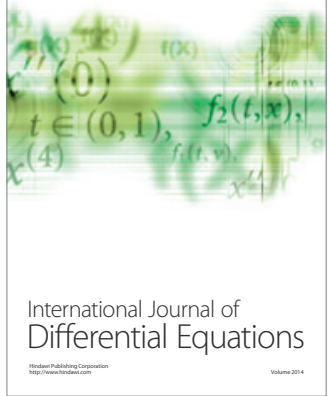
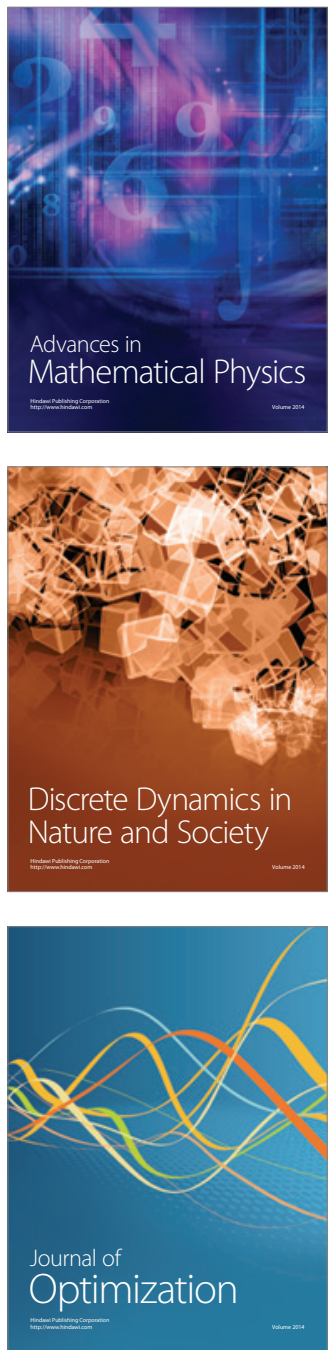